\title{
Inequalities for Hypergeometric Functions
}

\author{
By R. G. Buschman
}

\begin{abstract}
The upper and lower bounds for the determinant of a dominant diagonal matrix have been used recently to obtain bounds on the classical orthogonal polynomials. Similar methods are used here on the hypergeometric functions of Gauss and of Kummer.
\end{abstract}

Since our development will be with respect to the numerator parameter $a$, we shall use simply $F(a)$ to denote the hypergeometric function ${ }_{2} F_{1}(a, b ; c ; x)$. All of $a, b, c, x$ are considered positive, and we impose further restrictions as they appear in order to provide some sufficient conditions for the development. The technique is analogous to that used for the classical orthogonal polynomials by $\mathrm{H}$. M. Srivastava and J. L. Brenner [6]. If we replace $a$ by $a+n$ in the basic contiguous relation [3, $3.4(19)]$ and rearrange, we have

where

$$
F(a+n+1)=A_{n} F(a+n)+B_{n} F(a+n-1),
$$

$$
A_{n}=((b x-c) /(a+n)+2-x) /(1-x), \quad B_{n}=(c /(a+n)-1) /(1-x) .
$$

For $n \geqslant 0$, consider the $n+2$ by $n+2$ tridiagonal matrix

(1)

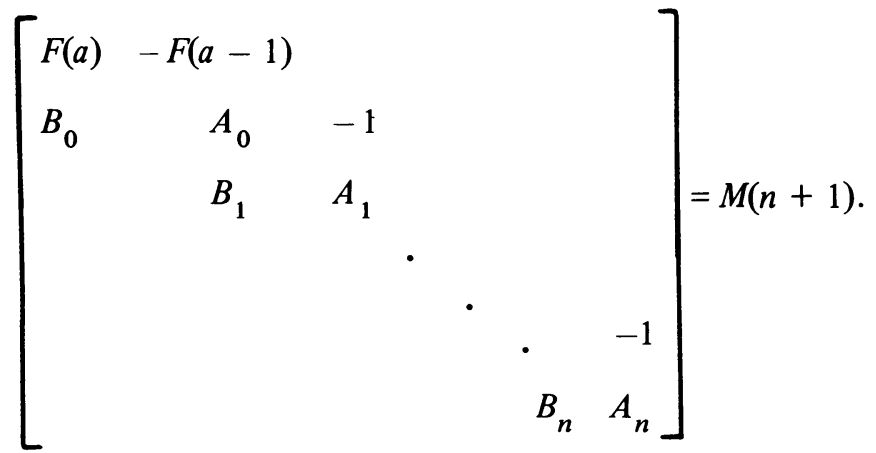

If we use the cofactor expansion of $\operatorname{det} M(n+1)$ with respect to the last row, it can be seen that $\operatorname{det} M(n+1)=F(a+n+1)$.

The matrix will have a strictly dominant diagonal provided that

$$
\begin{aligned}
|F(a)| & >|F(a-1)|, \\
\left|A_{k}\right| & >\left|B_{k}\right|+1, \quad \text { for } 0 \leqslant k<n, \\
\left|A_{n}\right| & >\left|B_{n}\right| .
\end{aligned}
$$

Received March 7, 1975; revised August 1, 1975.

AMS (MOS) subject classifications (1970). Primary 33A30; Secondary 15 A15, 33A40.

Key words and phrases. Gauss' hypergeometric function, Kummer's hypergeometric function, confluence principle, dominant diagonal matrix, modified Bessel function. 
From an examination of the power series representation of $F$ (with respect to $x$ ), we know that $\partial F(a) / \partial a>0$ for $a>0, x<1$; hence $a>1,0<x<1$ provide simple conditions for (2a) to be satisfied. Further, the condition $b>b x>2 c$ is sufficient for (2b) and (2c) to be satisfied. Thus, by the theorem of G. B. Price [4], upper and lower bounds for $F(a+n+1)$ are

$$
U=A_{n}(F(a)+F(a-1)) \prod_{k=0}^{n-1}\left(A_{k}+1\right),
$$

and

$$
L=A_{n}(F(a)-F(a-1)) \prod_{k=0}^{n-1}\left(A_{k}-1\right),
$$

respectively, where the absolute value symbols have been dropped because of our assumptions. (If the theorem of J. L. Brenner [1] is used, the lower bound can be improved; the formulae for the bound is then more complicated.)

The results can be summarized as follows, where the bounds (3U) and (3L) are used and the products are converted to $\Gamma$-functions.

THEOREM 1. If $a>1, b>b x>2 c>0$, then

$$
g(x) L<{ }_{2} F_{1}(a+n+1, b ; c ; x)<g(x) U,
$$

where

$$
\begin{aligned}
g(x) & =(1-x)^{-n-1}(b x-c+(2-x)(a+n)) \Gamma(a) / \Gamma(a+n+1), \\
U & =(F(a)+F(a-1))(3-2 x)^{n} \Gamma((b x-c) /(3-2 x)+a+n) \Gamma((b x-c) /(3-2 x)+a), \\
L & =(F(a)-F(a-1)) \Gamma(b x-c+a+n) / \Gamma(b x-c+a) .
\end{aligned}
$$

If the confluence principle $[3,3.5]$ is applied to the results of Theorem 1 , the analog for ${ }_{1} F_{1}$ is obtained.

THEOREM 2. If $a>1, x>2 c>0$, then

$$
g(x) L<{ }_{1} F_{1}(a+n+1 ; c ; x)<g(x) U,
$$

where

$$
\begin{aligned}
g(x) & =(x-c+2(a+n)) \Gamma(a) / \Gamma(a+n+1), \\
U & =\left({ }_{1} F_{1}(a ; c ; x)+{ }_{1} F_{1}(a-1 ; c ; x)\right) 3^{n} \Gamma((x-c) / 3+a+n) / \Gamma((x-3) / 3+a), \\
L & =\left({ }_{1} F_{1}(a ; c ; x)-{ }_{1} F_{1}(a-1 ; c ; x)\right) \Gamma(x-c+a+n) / \Gamma(x-c+a) .
\end{aligned}
$$

We note that the confluence principle cannot usefully be applied to the inequalities of T. M. Flett [2] to obtain information on ${ }_{1} F_{1}$. Further, the results of D. K. Ross, D. J. Bordelon [5] treat denominator parameters, but do not cover numerator parameters.

The analog of our process for the denominator parameter fails, since, although the reversed recurrence could be used to adjust for $F$ being a decreasing function of $c$, the dominant diagonal is not produced for interesting ranges of the parameters. The situation with respect to the numerator and denominator parameters of the confluent 
hypergeometric function $\psi(a ; c ; x)$ is reversed; we can obtain results for the denominator parameter.

THEOREM 3. If $c-1>a>0, x>0$, then

$$
L<x^{n} \psi(a ; c+n+1 ; x)<U,
$$

where

$$
\begin{aligned}
& U=(\psi(a ; c ; x)+\psi(a ; c-1 ; x))(c+n+1+x) \Gamma(c+n-1+2 x) / \Gamma(c+2 x), \\
& L=(\psi(a ; c ; x)-\psi(a ; c-1 ; x))(c+n-1+x) \Gamma(c+n-1) / \Gamma(c) .
\end{aligned}
$$

An analogous procedure can be applied to the modified Bessel function $K_{\nu}$.

THEOREM 4. If $v>1, v>x>0$, then

$$
L<(x / 2)^{n+1} K_{\nu+n+1}(x)<U
$$

where

$$
\begin{aligned}
& U=(\nu+n)\left(K_{\nu}(x)+K_{\nu-1}(x)\right) \Gamma(\nu+x / 2+n) / \Gamma(\nu+x / 2), \\
& L=(\nu+n)\left(K_{\nu}(x)-K_{\nu-1}(x)\right) \Gamma(\nu-x / 2+n) / \Gamma(\nu-x / 2) .
\end{aligned}
$$

Bounds for certain functions can be obtained by specializing the parameters of Theorems 1 and 2; analogous developments can be used for other functions satisfying three term-recurrence relations.

Department of Mathematics and Statistics University of Guelph

Guelph, Ontario, Canada NIG 2W1

1. J. L. BRENNER, “A bound for a determinant with dominant main diagonal," Proc. Amer. Math. Soc., v. 5, 1954, pp. 631-634. MR 16, 105.

2. T. M. FLETT, "Some inequalities for a hypergeometric integral," Proc. Edinburgh Math. Soc., v. (2) 18, 1972, pp. 31-34. MR $47 \# 5308$.

3. Y. L. LUKE, The Special Functions and Their Approximations. Vol. 1, Math. in Sci. and Engrg., vol. 53, Academic Press, New York, 1969. MR 39 \#3039.

4. G. B. PRICE, "Bounds for determinants with dominant principal diagonal," Proc. Amer. Math. Soc., v. 2, 1951, pp. 497-502. MR 12, 793.

5. D. K. ROSS \& D. J. BORDELON, "Inequalities for special functions," Problem 72-15, SIAM Rev., v. 15, 1973, pp. 665-670. MR 48 \#2437.

6. H. M. SRIVASTAVA \& J. L. BRENNER, "Bounds for Jacobi and related polynomials derivable by matrix methods," J. Approximation Theory, v. 12, 1974, pp. 372-377. 